\title{
Traffic Pattern based performance comparison of Reactive and Proactive protocols of Mobile Ad-hoc Networks
}

\author{
Vikas Singla, \\ Lecturer, \\ Department of Information Technology, \\ MIMIT, Malout, Punjab, India.
}

\author{
Parveen Kakkar \\ Sr. Lecturer, \\ Department of Computer Science \& Engg, \\ DAVIET, Jalandhar, Punjab,India.
}

\begin{abstract}
The primary objective of this research work is to study and investigate the performance measures of Reactive protocols (AODV, TORA) and Proactive protocols (DSDV) routing protocols of MANET using TCP \& CBR based traffic models. In this paper we will simulate the environment used for analyzing, evaluating and implementing AODV, DSDV and TORA routing protocols in MANET, to analyze the performance of above said protocols based on Packet Delivery Ratio, Average End-to-End Delay and Throughput. We will investigate the effect of change in number of nodes on MANET routing protocols. Here, we will analyze and compare the performance of MANET routing protocols based on both CBR and TCP based traffic patterns. We have used the NS-2 simulator for performing various simulations and used awk scripts for analyzing the results.
\end{abstract}

\section{Keywords}

MANET, Routing, Ad-hoc, Protocol, Performance, Simulation, AODV, TORA, DSDV.

\section{INTRODUCTION}

An ad-hoc network is a collection of wireless mobile nodes dynamically forming a temporary network without the aid of any established infrastructure or centralized administration. The system may operate in isolation, or may have gateways to interface with a fixed network. Ad hoc networks have no fixed routers; all nodes are capable of movement and can be connected dynamically in an arbitrary manner. Nodes of these networks, which function as routers, discover and maintain routes to other nodes in the network. The topology of the ad hoc network depends on the transmission power of the nodes and the location of the mobile nodes, which may change with time. The nodes (a router with multiple hosts and wireless communications devices) are free to move about and organize themselves randomly. These nodes may be located in or on airplanes, ships, trucks, cars, or on very small devices, and there may be multiple hosts per router. In general, Ad hoc wireless networks are self-creating, selforganizing, and self-administrating networks. Hence, they offer unique benefits and flexibility for a variety of situations and applications. Because of these features, the Ad hoc networks are used where wired network and mobile access is either unproductive or not feasible. A few possible examples include: earthquake hit areas, where infrastructure is destroyed, military soldiers in a destructive environment; virtual classrooms, biological detection, tracking of rare animal, space exploration, and undersea operations. A fundamental problem in ad hoc networking is how to deliver data packets among MNs efficiently without predetermined topology or centralized control, which is the main objective of ad hoc routing protocols. Since mobile ad hoc networks change their topology frequently, routing in such networks is a challenging task. Moreover, bandwidth, energy and physical security are limited. With the increasing popularity of mobile devices and wireless networks over the past few years, wireless ad-hoc networks has now become one of the most vibrant and active fields of communication and networking research.

\section{ROUTING PROTOCOLS}

Routing in Mobile Ad-hoc Network has been a subject of extensive research over the past several years. Because of the fact that it may be necessary to pass several hops (multi-hop) before a packet reaches the destination, a routing protocol is needed. Routing protocol has two functions:

i. Selection of routes for various source-destination pairs

ii. Delivery of messages to their correct destination.

The second function is conceptually straightforward using a variety of protocols and data structures (routing tables).

Ad-hoc routing protocols can be classified based on different criteria. Depending upon the routing mechanism employed by a given protocol, it may fall under more than one class. Routing protocols for Ad-hoc networking can be classified into four categories viz. (i) Based on routing information update routing mechanism (proactive or table-driven, reactive or on-demand and hybrid protocols), (ii) Based on the use of Temporal information (Past Temporal and Future Temporal) for routing, (iii) Based on routing topology (Flat Topology, Hierarchical Topology), (iv) Based on the Utilization of Specific Resources (Power Aware Routing and Geographical Information Assisted Routing) .

\subsection{Adhoc On-Demand Distance Vector (AODV) $[4,5,6,7]$}

AODV is a purely reactive routing protocol. In this protocol, each terminal does not need to keep a view of the whole network or a route to every other terminal. Nor does it need to periodically exchange route information with the neighbor terminals. Furthermore, only when a mobile terminal has packets to send to a destination does it need to discover and maintain a route to that destination terminal. In AODV, each terminal contains a route table for a destination. A route table stores the following information: destination address and its sequence number, active neighbors for the route, hop count to the destination, and expiration time for the table. The expiration time is updated each time the route is used. If this route has not been used for a specified period of time, it is discarded.

\subsection{Destination Sequenced Distance-Vector Routing (DSDV) [8]}

The Destination Sequenced Distance Vector Protocol (DSDV) is a proactive, distance vector protocol which uses the Bellmann - 
Ford algorithm. DSDV is a hop-by-hop distance vector routing protocol, wherein each node maintains a routing table listing the "next hop" and "number of hops" for each reachable destination. This protocol requires each mobile station to advertise, to each of its current neighbors, its own routing table (for instance, by broadcasting its entries). The entries in this list may change fairly dynamically over time, so the advertisement must be made often enough to ensure that every mobile computer can almost always locate every other mobile computer of the collection. In addition, each mobile computer agrees to relay data packets to other computers upon request. This agreement places a premium on the ability to determine the shortest number of hops for a route to a destination we would like to avoid unnecessarily disturbing mobile hosts if they are in sleep mode. In this way a mobile computer may exchange data with any other mobile computer in the group even if the target of the data is not within range for direct communication.

\subsection{Temporary Ordered Routing Algorithm (TORA)}

The Temporally Ordered Routing Algorithm (TORA) is a highly adaptive, efficient and scalable distributed routing algorithm based on the concept of link reversal . TORA is proposed for highly dynamic mobile, multi-hop wireless networks. It is a source-initiated on-demand routing Protocol . It finds multiple routes from a source node to a destination node. The main feature of TORA is that the control messages are localized to a very small set of nodes near the occurrence of a topological change. To achieve this, the nodes maintain routing information about adjacent nodes. The protocol has three basic functions: Route creation, Route maintenance and Route erasure. TORA can suffer from unbounded worst-case convergence time for very stressful scenarios. TORA has a unique feature of maintaining multiple routes to the destination so that topological changes do not require any reaction at all. The protocol reacts only when all routes to the destination are lost. In the event of network partitions the protocol is able to detect the partition and erase all invalid routes.

\section{PROBLEM FORMULATION $[23,24,26]$}

The IETF MANET working group mandate was to standardize IP routing protocols in MANETs. The RFC 2501 specifies the charter for the working group. The RFCs still has unanswered questions concerning either implementation or deployment of the protocols. Nevertheless, the working group identifies the proposed algorithms as a trial technology. Aggressive research in this area has continued since then, with prominent studies on routing protocols such as Ad hoc On-demand Distance Vector (AODV), Destination-Sequenced Distance-Vector Routing protocol (DSDV) and Dynamic Source Routing Protocol (DSR). Several studies have been done on the performance evaluation of routing protocols based on CBR traffic pattern using different evaluation methods. Different methods and simulation environments give different results and consequently, there is need to broaden the spectrum to account for effects not taken into consideration in a particular environment.

It is observed that most of the research work is based on CBR traffic pattern whereas most of the traffic approximately $95 \%$ on the Internet carries TCP. It is desirable to study and investigate the performance of different MANET routing protocols under both CBR and TCP traffic patterns. In this paper, we will evaluate the performance of Reactive protocols (AODV and TORA) and
Proactive protocols (DSDV) of mobile ad-hoc network routing protocols for both CBR and TCP traffic patterns. The performance of these routing protocols is evaluated with respect to various parameters such as average end-to-end delay, throughput and packet delivery ratio.

\section{PERFORMANCE METRICS [2,23]}

Mobile ad hoc networks have several inherent characteristics (e.g. dynamic topology, time-varying and bandwidth constrained wireless channels, multi-hop routing, and distributed control and management). Design and performance analysis of routing protocols used for mobile ad hoc network (MANET) is currently an active area of research. To judge the merit of a routing protocol, one needs metrics - both qualitative and quantitative-with which to measure its suitability and performance. Specifically, this paper evaluates the performance comparison of AODV, DSDV and TORA routing protocols on the following performance metrics: Average end-to-end delay, Packet delivery ratio and throughput

\subsection{Packet Delivery Ratio}

Packet delivery ratio is calculated by dividing the number of packets received by the destination through the number of packets originated by the application layer of the source. It specifies the packet loss rate, which limits the maximum throughput of the network. The better the delivery ratio, the more complete and correct is the routing protocol.

\subsection{Average End-To-End Delay}

Average End-to-End delay (seconds) is the average time it takes a data packet to reach the destination. This metric is calculated by subtracting "time at which first packet was transmitted by source" from "time at which first data packet arrived to destination". This includes all possible delays caused by buffering during route discovery latency, queuing at the interface queue, retransmission delays at the MAC, propagation and transfer times. This metric is significant in understanding the delay introduced by path discovery.

\subsection{Throughput}

The throughput of the protocols can be defined as percentage of the packets received by the destination among the packets sent by the source. It is the amount of data per time unit that is delivered from one node to another via a communication link. The throughput is measured in bits per second (bit/s or bps).

\section{SIMULATION AND PERFORMANCE ANALYSIS [24]}

In this paper, we have taken two different scenarios. In the first scenario, traffic pattern is taken as CBR and no. of nodes have been varied and performance comparisons has been made between AODV, DSDV and TORA protocols. In the second scenario, traffic pattern is taken as TCP and no. of nodes have been varied and performance comparisons has been made between AODV, DSDV and TORA protocols. Identical mobility pattern are used across protocols to gather fair results.

\subsection{Test Scenario 1}

In the first scenario, We have chosen the simulation based on CBR traffic pattern. Parameters of this scenario are summarized in table 5.1. CBR sources are used that started at different times 
because we want to get a general view of how routing protocol behaves.

Table 5.1: Simulation Parameters for Test Scenario 1

\begin{tabular}{|l|l|}
\hline \multicolumn{1}{|c|}{ Parameter } & \multicolumn{1}{c|}{ Value } \\
\hline Number of nodes & $25,50,75,100$ \\
\hline Maximum Speed & $20 \mathrm{~m} / \mathrm{s}$ \\
\hline Simulation time & $50 \mathrm{sec}$ \\
\hline Pause Time & $5 \mathrm{sec}$ \\
\hline Environment Size & $1000 \times 1000$ \\
\hline Packet Size & 512 bytes \\
\hline Traffic Type & CBR \\
\hline Packet Rate & 4 packet/sec \\
\hline
\end{tabular}

\subsection{Test Scenario 2}

In the second scenario, We have chosen the simulation based on TCP traffic pattern. Parameters of this scenario are summarized in table 5.2. Here, TCP sources are used which use flow control and retransmission feature.

Table 5.2: Simulation Parameters for Test Scenario 2

\begin{tabular}{|l|l|}
\hline \multicolumn{1}{|c|}{ Parameter } & \multicolumn{1}{c|}{ Value } \\
\hline Number of nodes & $25,50,75,100$ \\
\hline Maximum Speed & $20 \mathrm{~m} / \mathrm{s}$ \\
\hline Simulation time & $50 \mathrm{sec}$ \\
\hline Pause Time & $5 \mathrm{sec}$ \\
\hline Environment Size & $1000 \times 1000$ \\
\hline Packet Size & 512 bytes \\
\hline Traffic Type & TCP \\
\hline Packet Rate & 4 packet/sec \\
\hline
\end{tabular}

\section{RESULTS AND PERFORMANCE COMPARISION}

Performance of AODV, TORA and DSDV protocols is evaluated under both CBR and TCP traffic pattern. Extensive Simulation is done by using NS-2.

\subsection{Average end-to-end Delay}

Average end-to-end Delay of proactive routing protocols (DSDV) is less as compared to reactive routing protocols (AODV and TORA) in any kind of traffic pattern i.e. either CBR (Figure1) or TCP (Figure2). Average end-to-end Delay is also remains almost constant in DSDV whereas it varies in the case of AODV and TORA protocols with respect to change in number of nodes.

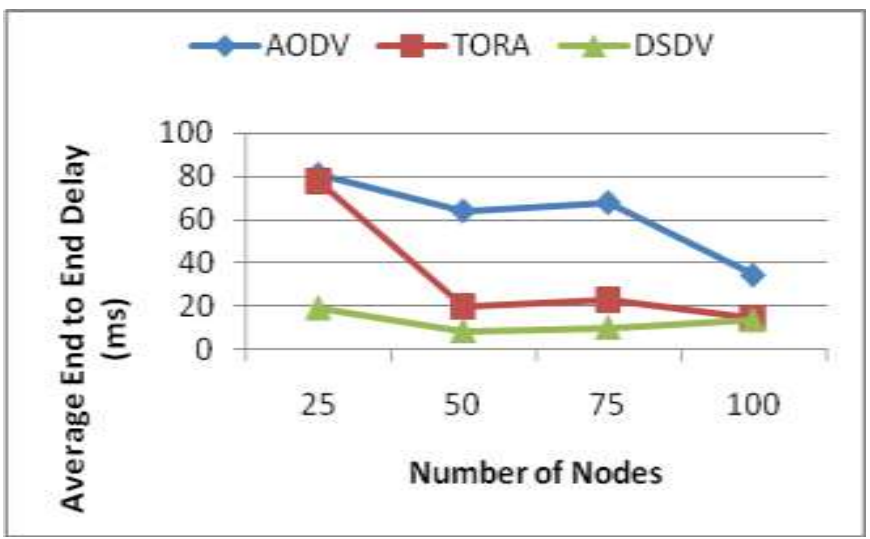

Figure 1 Average End to End Delay for CBR Traffic Pattern

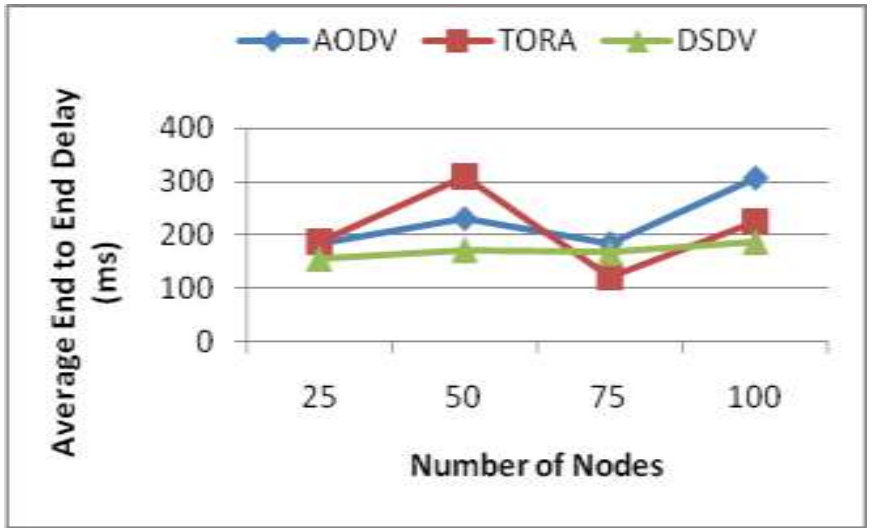

Figure 2 Average End to End Delay TCP Traffic Pattern

\subsection{Packet Delivery Ratio}

In case of CBR traffic Reactive protocols deliver almost all the originated data packets converging to $100 \%$ delivery whereas Proactive protocols(DSDV) Packet Delivery Ratio is approx 50\% (Figure 3). Reactive protocols perform better than the proactive protocols in case of CBR traffic pattern. In the case of TCP traffic pattern (Figure 4), Packet delivery ratio of AODV protocols remains almost constant whereas it changes rapidly for TORA and DSDV protocols irrespective of the network load.

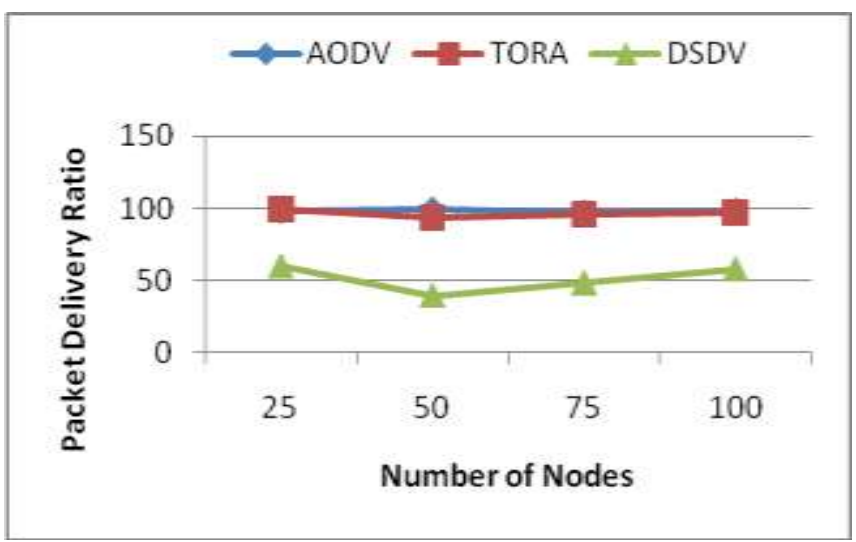

Figure 3 Packet Delivery Ratio for CBR Traffic Pattern 


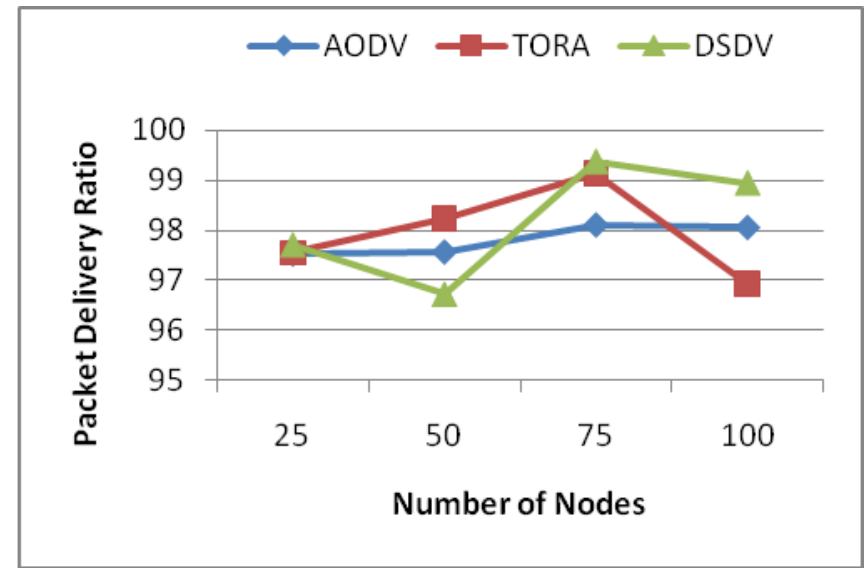

Figure 4 Packet Delivery Ratio for TCP Traffic Pattern

\subsection{Throughput}

In case of CBR traffic pattern throughput of AODV and TORA protocols is almost same and is better than as compared to DSDV protocols. In case of CBR traffic, throughput remains almost constant for all three protocols irrespective of number of nodes. In case of TCP traffic, throughput changes rapidly with respect to change in the number of nodes.

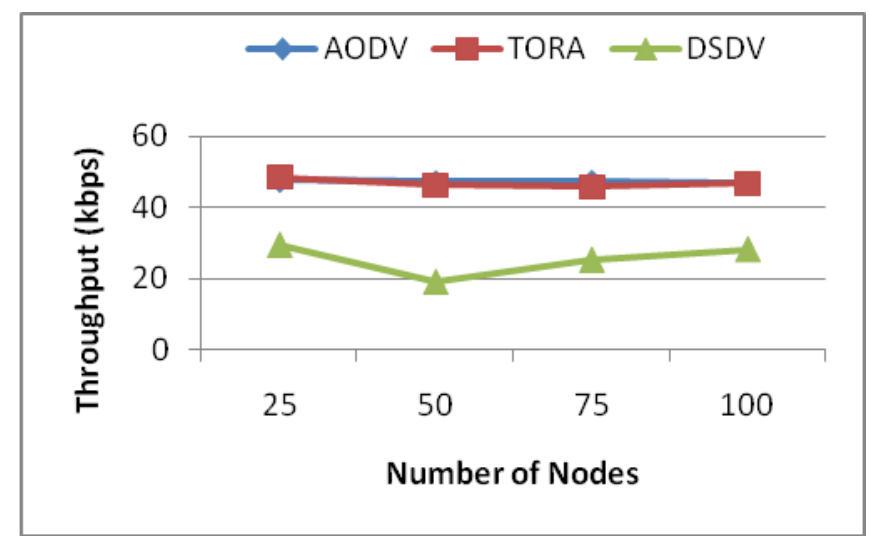

Figure 5 Throughput for CBR Traffic Pattern

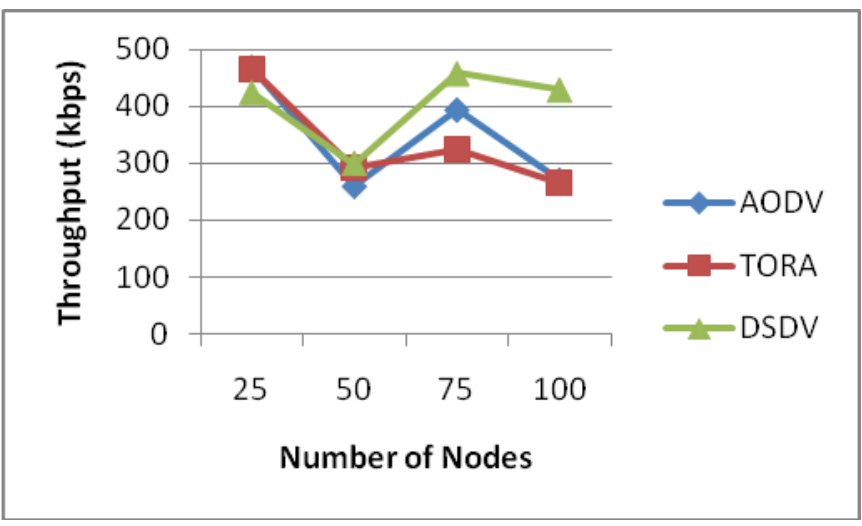

Figure 6 Throughput for TCP Traffic Pattern

\section{CONCLUSIONS \& FUTURE WORK}

This study was conducted to evaluate the performance Reactive (AODV , TORA) and Proactive protocols (DSDV) of MANET based on both CBR and TCP traffic. These routing protocols were compared in terms of Packet delivery ratio, Average end-toend delay and Throughput when subjected to change in no. of nodes. Simulation results show that Reactive protocols better in terms of packet delivery ratio and average end-to-end delay Future work will be to evaluate the performance of these protocols by varying the speed, pause time. Performance can also be analyzed for other parameters like Jitter, Routing Overhead. By evaluating the performance of these protocols new protocols can be implemented or changes can be suggested in the earlier protocols to improve the performance.

\section{REFERENCES}

[1] Das S. R., Perkins C. E., Royer E. M. and Marina M. K., "Performance comparison of two on-demand routing protocols for ad hoc networks," IEEE Personal Communications Magazine, special issue on Mobile Ad Hoc Networks, vol. 8, no. 1, pp. 16-29, February 2001.

[2] Dyer T. D., Boppana R. V., "A Comparison of TCP performance over three routing protocols for mobile adhoc networks", ACM Symposium on Mobile Adhoc Networking \& Computing (Mobihoc), October 2001.

[3] Wang F., Zhang Y., “ Improving TCP performance over Mobile Adhoc Networks with out of order detection and response", (C) ACM Mobihoc, June 2002.

[4] Clausen T., Jacquet P., Viennot L., "Comparative study of CBR and TCP performance of MANET routing protocols", Project HiPERCOM - INRIA, France, 2003.

[5] Mani P., Petr D.W., "Development and performance characterization of enhanced AODV routing for CBR and TCP traffic", (C) 2004 IEEE.

[6] Ahmed S. and Alam M. S., "Performance Evaluation of Important Ad Hoc Network Protocols", Hindawi Publishing Corporation, EURASIP Journal on Wireless Communications and Networking Volume 2006, Article ID 78645, Pages 1-11.

[7] Grew P., Giudici F., Pagani E., "Specification of a functional architecture for e-learning supported by wireless technologies", Pervasive Computing and Communications Workshops, 2006. PerCom Workshops 2006. Fourth Annual IEEE International Conference on Volume, Issue, 13-17 March 2006 Page(s):5 pp. - 220

[8] Hogie L., Bouvry P., "An overview of MANETs Simulation", Electronic notes in theoretical computer science, (C) 2006 Elsevier, doi:10.1016/j.entcs.2005.12.025.

[9] Lakshmi M. and Sankaranarayanan P.E., "Performance analysis of three routing protocols in wireless ad hoc networks", Information technology Journal 5 (1): 114-120, () 2006 Asian Network for Scientific Information.

[10] Sundaram Rajagopalan and Chein-Chung Shen, "What does Using TCP as an Evaluation Tool Reveal about MANET Routing Protocols?", IWCMC'06 C 2006 ACM Journal.

[11] Zaballos A., Vallejo A., Corral G., Abe.lla J., "AdHoc routing performance study using OPNET Modeler", 
OPNETWORK'2006, Washington DC (United States), August 2006.

[12] Alsaadi M.Y., Qian Y.," Performance Study of a Secure Routing Protocol in Wireless Mobile Ad Hoc Networks", Wireless Pervasive Computing, 2007. ISWPC apos; 07. 2nd International Symposium on Volume, Issue, 5-7 Feb. 2007 Page(s): - Digital Object Identifier 10.1109/ISWPC.2007.342641, 1-4244-0523-8/07/\$20.00 (C)2007 IEEE.

[13] Jayakumar G. and Gopinath G., "Ad Hoc Mobile Wireless Networks Routing Protocols- A Review," Journal of Computer Science, Vol. 3, No.8, pp. 574-582, 2007.

[14] Layuan L., Chunlin L., Peiyan L., "Performance evaluation and simulations of routing protocols, Computer Communications 30 (2007) 1890-1898, (C2007 Elsevier B.V.

[15] Trung H. D. , Benjapolakul W., Duc P. M., "Performance evaluation and comparison of different ad hoc routing protocols", Computer Communications 30 (2007) 2478-2496 (C) 2007 Elsevier.

[16] Werapun W., Thavornvisit K., "Performance comparison of TCP and CBR in MAODV adhoc network", Systems and Networks Communications ICSNC @ C IEEE, August 2007.

[17] Jayakumar G. and Gopinath G., "Performance Comparison of Two On-demand Routing Protocols for Ad-hoc Networks based on Random Way Point Mobility Model" , American Journal of Applied Sciences 5 (6): 659-664, (C) 2008 Science Publications.

[18] Masoudifar M., "A review and performance comparison of QoS multicastrouting protocols for MANETs", Ad Hoc Networks 7 (2009) 1150-1155, doi: 10.1016/j.adhoc.2008.10.004, 2008 Elsevier B.V.
[19] Mukhija R., Saluja R., "Performance comparison of ad-hoc network routing protocols in different sizes", MATEIT : 169-176, 2008.

[20] Aziz S. R. A., Endut N. A., Abdullah S. and Daud M. N. M., "Performance evaluation of AODV, DSR and DYMO routing protocol in MANET", CSSR 08-09, 14 - 15 March 2009.

[21] Fall K., Varadhan K., "The ns manual, The VINT Project", 2009. Available from: <http://www.isi.edu/nsnam/ns/doc/nsdocumentation.html $>$.

[22] Huang R., Zhuang Y., Cao Q., "Simulation and Analysis of Protocols in Ad Hoc Network", International Conference on Electronic Computer Technology @ 2009 IEEE.

[23] Suresh Kumar, R K Rathy and Diwakar Pandey, "Traffic pattern based performance comparison of two reactive routing protocols for ad hoc networks using NS@”, (C) 2009 IEEE.

[24] Vikas Singla, Rakesh Singla and Ajay Kumar, "Performance Evaluation and Simulation of Mobile Ad-hoc Network Routing Protocols", International Journal of Engineering and Information Technology, Volume 1 No. 1 October 2009.

[25] Qamar S., Manoj K., "Impact of Random Loss on TCP Performance in Mobile Ad-hoc Networks (IEEE 802.11): A Simulation-Based Analysis", International Journal of Computer Science and Information Security, Vol. 7, No. 1, 2010, pno. 228-233.

[26] Yogesh Chaba, Yudhvir Singh, Manish Joon, "Simulation Based Performance Analysis of On-Demand Routing Protocols in MANETs," Second International Conference on Computer Modeling and Simulation, 2010. 